\title{
Implications of Geodetic Strain Rate for Future Earthquakes, With a Five- Year Forecast of M5 Earthquakes in Southern California
}

\author{
Zheng-Kang Shen, David D. Jackson, and Yan Y. Kagan \\ Department of Earth and Space Sciences \\ University of California \\ Los Angeles, CA 90095-1567
}

\begin{abstract}
$\underline{\text { Abstract }}$
We construct an earthquake likelihood model based on the hypothesis that earthquake frequency and magnitude distribution are related to geodetic strain rate in two ways: a) seismicity rate is steady and proportional to the average horizontal maximum shear strain rate during interseismic time period between large earthquakes; and b) earthquake magnitude distribution is spatially invariant except for an amplitude constant which is proportional to the maximum horizontal shear strain rate. We use geodetically determined maximum shear strain rate averaged from 1993-2001 to "forecast" earthquakes of $M \geq 5$, and compare strain rate with the earthquake occurrence rate. In a preliminary test, there were few earthquakes within the top $10 \%$ of the area with highest strain rate, but most of the earthquakes did occur in the next $15 \%$ of the region with highest strain rate. About $75 \%$ of the earthquakes occurred at $25 \%$ area with highest strain rate. Therefore our preliminary test of the method yields a cautiously promising result. A new forecast for the next 5 years starting January 1, 2006 is presented.
\end{abstract}

\section{$\underline{\text { Introduction }}$}

Despite great efforts made by geoscientists, precise earthquake prediction still remains illusive. It has become a growing consensus in the recent years that the earthquake process is partially stochastic, and it is more appropriate to take a probabilistic approach to forecast earthquakes. Because the inter-, pre-, and post-seismic processes are considered to be mechanically different, different stochastic models may have to be adopted for earthquake forecast models during these time periods in an earthquake cycle. In this study we attempt to develop a probabilistic earthquake forecast model of intermediate to long times during the inter-seismic time period, constrained by geodetically derived crustal strain rates.

Earthquakes take place along faults that rupture under stress and release elastic energy accumulated in the neighborhood over the inter-seismic time period. Active faults are weak zones in the crust and tend to accumulate higher strains than the surrounding regions. Therefore regions with higher strain concentration are often the locations of seismogenic faults, and more prone to be the source of future earthquakes. From the Kostrov formula (Kostrov, 1974) we know that the seismic moment release on a fault is proportional to the slip. The detailed conditions that result in earthquakes may be very complicated, but over a large region and long time scale, plate tectonic theory implies that, except at some creeping plate boundaries, the 
tectonic moment increase should equal the moment release in earthquakes. Kostrov's formula expresses that moment balance, and it may hold only in a statistical sense as we consider smaller regions and shorter time intervals. An implication of Kostrov's formula is that the earthquake rate should be related to strain rate, rather than strain itself. At higher resolution, the actual strain level and other variables may also be important. In any case we adopt the hypothesis that earthquake rate is proportional to strain rate, and proceed to test that hypothesis against future earthquake occurrence. This hypothesis may not be valid, however, if a large earthquake occurs and reset the stress field for the region. Our first assumption, therefore, is that seismicity rate is steady and proportional to the average horizontal maximum shear strain rate during interseismic time periods between large earthquakes. Our second assumption is that the shape of the earthquake magnitude distribution is spatially invariant and follows a tapered GutenbergRichter relationship derived by Bird and Kagan (2004) for the continental transform fault environment, but the amplitude distribution varies spatially and is proportional to the maximum horizontal shear strain rate. The earthquake probability at a given spot $x$ and magnitude $M$ is therefore described as:

$$
P(x, M)=A(x) F(M)
$$

where $A(x)$ is proportional to the maximum shear strain rate field, and

$$
F(M)=10^{-1.5 \beta(M-M m i n)} \exp \left[10^{1.5(M \min -M c)}-10^{1.5(M-M c)}\right]
$$

which is the tapered Gutenberg-Richter magnitude distribution for the events occurred around the continental transform fault environment proposed by Bird and Kagan (2004), with the corner magnitude $M_{c}=8.02$, and the exponential falloff rate for the seismic moment distribution $\beta=$ 0.65 . Minimum earthquake magnitude $M_{\min }$ is 4.95 for this study. This forecast model, as mentioned in the first assumption, is related to mean strain rate derived during an interseismic time period between large earthquakes.

\section{Geodetic Strain Rate Estimation}

The geodetic strain rate in southern California is used to infer earthquake potential, and is derived by interpolating the velocity field from the Southern California Crustal Motion Map version 3.0 (CMM3, http://epicenter.usc.edu/cmm3). The CMM3 station velocities were derived from geodetic data spanning a time period 1970-2001, with individual sites observed at various epochs. To forecast future earthquakes we need to select data representing the present deformation rate as closely as possible. This is realized by screening out the stations whose average observation epochs occur before 1993.0. In doing so we assure that the data were collected primarily after the $1992 \mathrm{Mw} 7.3$ Landers earthquake, which has been the predominant source of recent transient deformation in southern California (Shen and Zeng, 2005). GPS data collected post and in the epicentral area of the $1999 \mathrm{Mw} 7.1$ Hector Mine earthquake were removed from the CMM3 data set, thus the geodetic data set used to derive the strain rate field in this study can be regarded as a post-Landers deformation field, and used to compare with the seismicity pattern post 1993 . 
Interpolation of the station velocities is done through a series of regressions, each time estimating strain and rotation rates at one spot using velocity data in the neighborhood (Shen et al., 1996; Jackson et al., 1997). A locally uniform strain rate field is assumed in the process, and the velocity data are reweighted by a Gaussian function $\exp \left(-\Delta^{2} / \sigma^{2}\right)$, where $\Delta$ is the distance between a geodetic station and the spot being evaluated, and $\sigma$ is a smoothing distance that is determined optimally through balancing a trade-off between the formal uncertainty estimate of the strain rate and the total weight assigned to the data. We obtain $\sigma$ ranging between 20 and $150 \mathrm{~km}$, with a typical value of $\sim 40 \mathrm{~km}$. The derived maximum shear strain rate is shown in Fig. 1a. The estimated region covers all of the southern California up to $37^{\circ} \mathrm{N}$ latitude except at the northwest corner, where there are insufficient data to estimate the strain rate reliably. GPS data observed at the Coso and Obsidian Buttes geothermal areas were removed in the CMM3 solution, thus local deformation resulted from geothermal activities should have negligible effect in this strain rate field. The high strain rate along the San Andreas north of $36^{\circ} \mathrm{N}$ latitude is partially influenced by fault creep. This potentially will affect our forecast result since the strain rate field is not completely elastic and thus seismogenic. However, because the effect is local and the affected region quite small, we do not expect our neglect of fault creep to produce a large error.

\section{$\underline{\text { Retrospective "Forecast" Test and Future Earthquake Forecasts }}$}

Fig. 1a shows the contour map of the present day maximum shear strain rate, and the spatial distribution of $\mathrm{M} \geq 5.0$ earthquakes observed from 1950-2005. The earthquake locations are taken from the "point-source" catalog developed Kagan et al. (2006). Fig. 1b shows cumulative histograms of the corresponding shear strain rate and earthquake count, with unit areas sorted in decreasing order of strain rate. Our first assumption states that the two curves should match each other. The strain rates, however, will lose much of the predictive power if the earthquake count curve is significantly below the strain rate curve. They, on the other hand, will have more predictive power (e.g. more accurate prediction of earthquake locations) if the earthquake count curve is significantly above the strain rate courve. Fig. 1b shows the spatial concentration of earthquakes versus geodetically derived strain rate, which is similar to Figure 4 of Helmstetter et al. (this issue), with the exception that the strain rate was derived seismologically in Helmstetter et al. than geodetically. It is evident from Fig. $1 \mathrm{~b}$ that the spatial concentration of earthquakes matches the strain concentration quite well, about $75 \%$ of the events occurred within $25 \%$ of the area with highest strain rate, seemingly representing a successful retrospective test of the first assumption. However, this exercise cannot be a real test, because most of the earthquakes predated the strain measurements, and we know that the high strain rate at some regions resulted from, rather than preceded, past earthquakes.

We next use the shear strain rate to forecast earthquakes from 1993.0-2005.5 (Fig. 2a). This is a much stronger test because the coseismic and postseismic effects of the 1999 Hector Mine earthquake have been removed from the data, and the strain rate field should reflect the steady deformation in most of southern California. The result (Fig. 2b) shows that although the agreement between geodetically estimated earthquake potential and actual earthquake occurrence is not as good for the areas with highest (top 10\%) strain rate as that shown in Fig. 1b, most of the earthquakes did occur in next $15 \%$ of the region with highest strain rate. As revealed in Fig. $2 \mathrm{~b}$, again $\sim 75 \%$ of the earthquakes occurred within $\sim 25 \%$ of the area with highest strain rate. Therefore our preliminary test of the method yields a cautiously promising result. 
Using this approach we have made new forecasts for the probability of the $M \geq 5$ earthquakes in southern California for the next 5 years. The forecasts are for earthquakes of two categories: one for all the events and the other for de-clustered (meaning aftershocks excluded). The two forecasts are the same in terms of the spatial and magnitude distribution pattern except the scaling factors, which are calibrated using the earthquake catalogs from Kagan et al. (2006). Specifications of the forecasts can be found at

http://scec.ess.ucla.edu/ zshen/eqprb/eqprb.html.

\section{$\underline{\text { Discussions }}$}

Our forecasts are based on geodetically observed strain rate averaged over a time period of about a decade. From now on till the next large earthquake, the deformation rate in southern California is expected to be more or less the same as what we have obtained. Therefore if the earthquake occurrence rate is proportional to the strain rate as we have assumed, the strain rate, in principal, could be a better intermediate term predictor of earthquakes than other predictors based on historical earthquake and/or geological information. This is because that the historical seismological and geologic predictors are devised based on information of fault slip/seismic moment release rates averaged over hundreds to thousands of years, and perhaps reflect more the long term, less the intermediate term behavior of earthquake occurrence. On the other hand, geodetically observed strain rate has its own limitations when used for earthquake forecasting. For example, geodetically observed strains include both elastic and anelastic strains, and in many cases it is difficult to differentiate the two without a priori knowledge. Because only the elastic strain is responsible for earthquakes, forecasts using geodetic information contain errors, particularly across faults which are creeping and in regions where significant amount of deformation take place plastically. Seismic and geologic observations are pretty much immune to such a problem, and can be important supplement to geodetic information to be used for earthquake forecast.

Acknowledgments. We thank Sue Hough and an anonymous reviewer for their constructive comments. The work has been partially supported by the Southern California Earthquake Center (SCEC). SCEC is funded by NSF Cooperative Agreement EAR-0106924 and USGS Cooperative Agreement 02HQAG0008. The SCEC publication number for this paper is 945. 


\section{$\underline{\text { References }}$}

Bird, P, and Y. Kagan. Plate-tectonic analysis of shallow seismicity; apparent boundary width, beta, corner magnitude, coupled lithosphere thickness, and coupling in seven tectonic settings, Bull. Seismol. Soc. Am., 94, no.6, 2380-2399, 2004.

Helmstetter, A., Y. Y. Kagan, and D. D. Jackson, Time-independent earthquake forecasts for California based on smoothed seismicity, Seismol. Res. Lett., submitted (this issue), 2006.

Jackson, D. D., Z.-K. Shen, D. Potter, X. Ge, L. Sung, Earthquakes and strain in southern California, Science, 277, 1621-1622, 1997.

Kagan, Y. Y., D. D. Jackson, and Y. F. Rong, A new catalog of southern California earthquakes, 1800-2005, Seismol. Res. Lett., 77(1), 30-38, 2006.

Kostrov, B. V., Seismic moment and energy of earthquakes, and seismic flow of rock, Izvestiya Academy of Sciences of the USSR (Physics of Solid Earth), 1, 23-40, 1974.

Shen, Z.-K., X. B. Ge, D. D. Jackson, D. Potter, M. Cline, and L. Sung, Northridge earthquake rupture models based on the global positioning system measurements, Bull. Seismol. Soc. Am., 86, 1B, S37-S48, 1996.

Shen, Z.-K., and Y. Zeng, Geodetically observed temporal variation of crustal deformation in southern California and northern rim of Tibet (abstract), EOS Trans. AGU, 86, Fall Meeting, G51D-05, 2005.

\section{Figure captions}

Figure 1. a) Maximum shear strain rate and earthquakes. Color contours show geodetically derived maximum shear strain rate at nano-radian/yr. Earthquakes of $M \geq 5$ occurred 1950-2005 are shown as white circles, with the size of the circles proportional to the magnitude. b) Cumulative histograms of shear strain rate integrated over area (black curve) and earthquake count (blue curve, totaling 168 events). The shear strain rate is sorted in decreasing order, with the highest strain rate positioned to the left of the diagram. The vertical bar marks $25 \%$ of the total area measured from origin.

Figure 2. The same as Figure 1 except that the earthquakes were 31 events of $M \geq 5$ in total occurred 1993-2005. 
Figure 1a

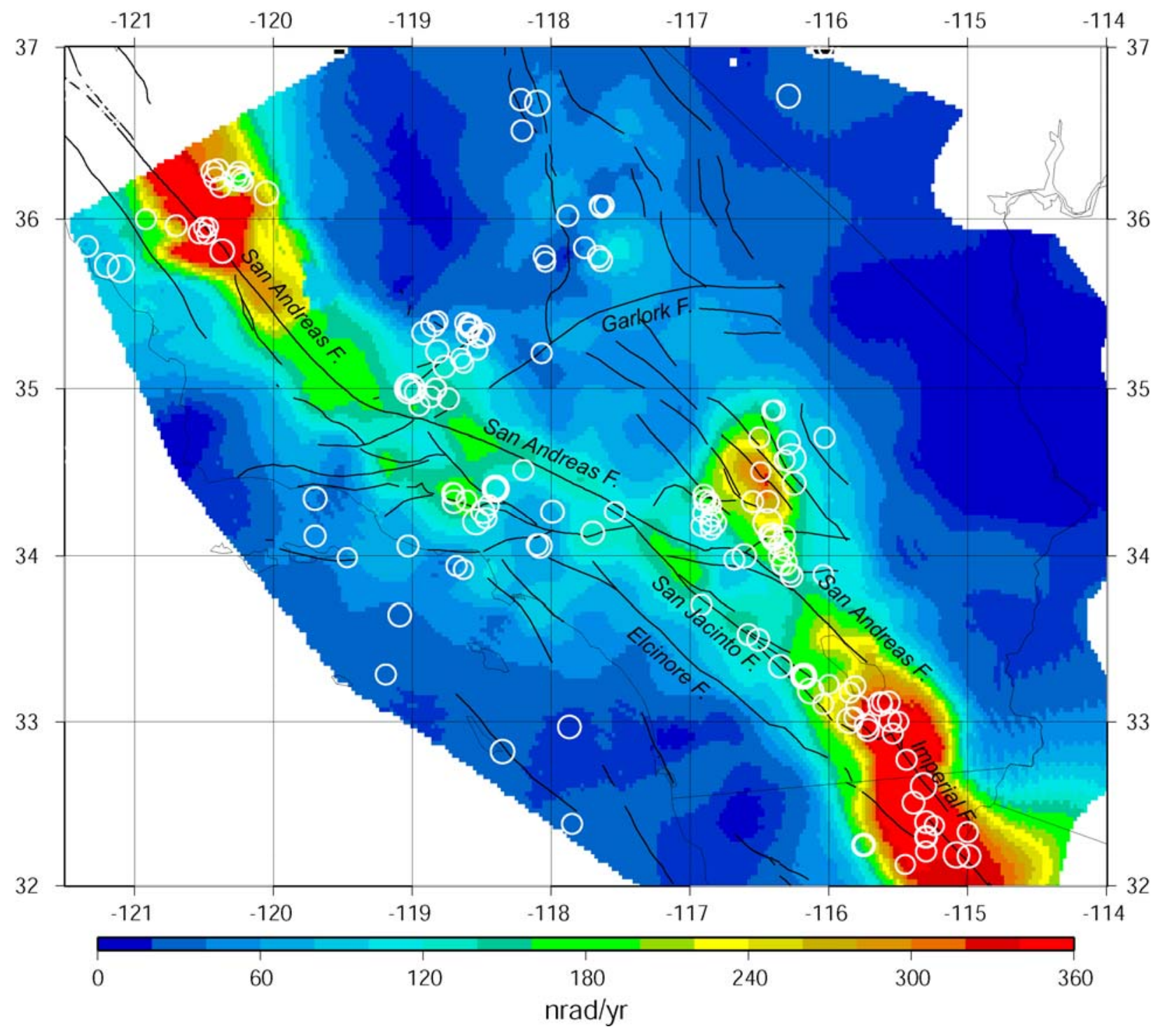


Figure 1b

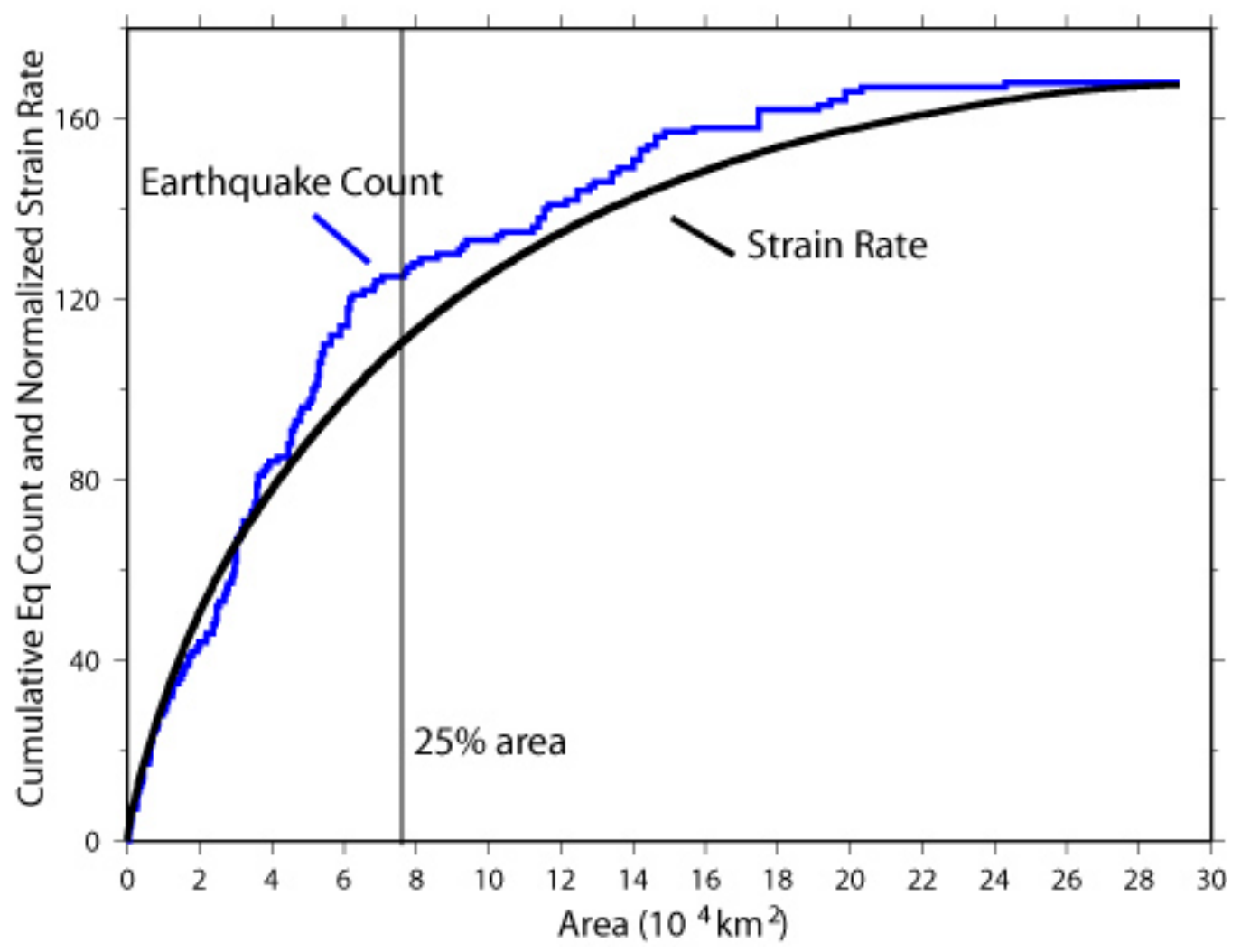


Figure 2a

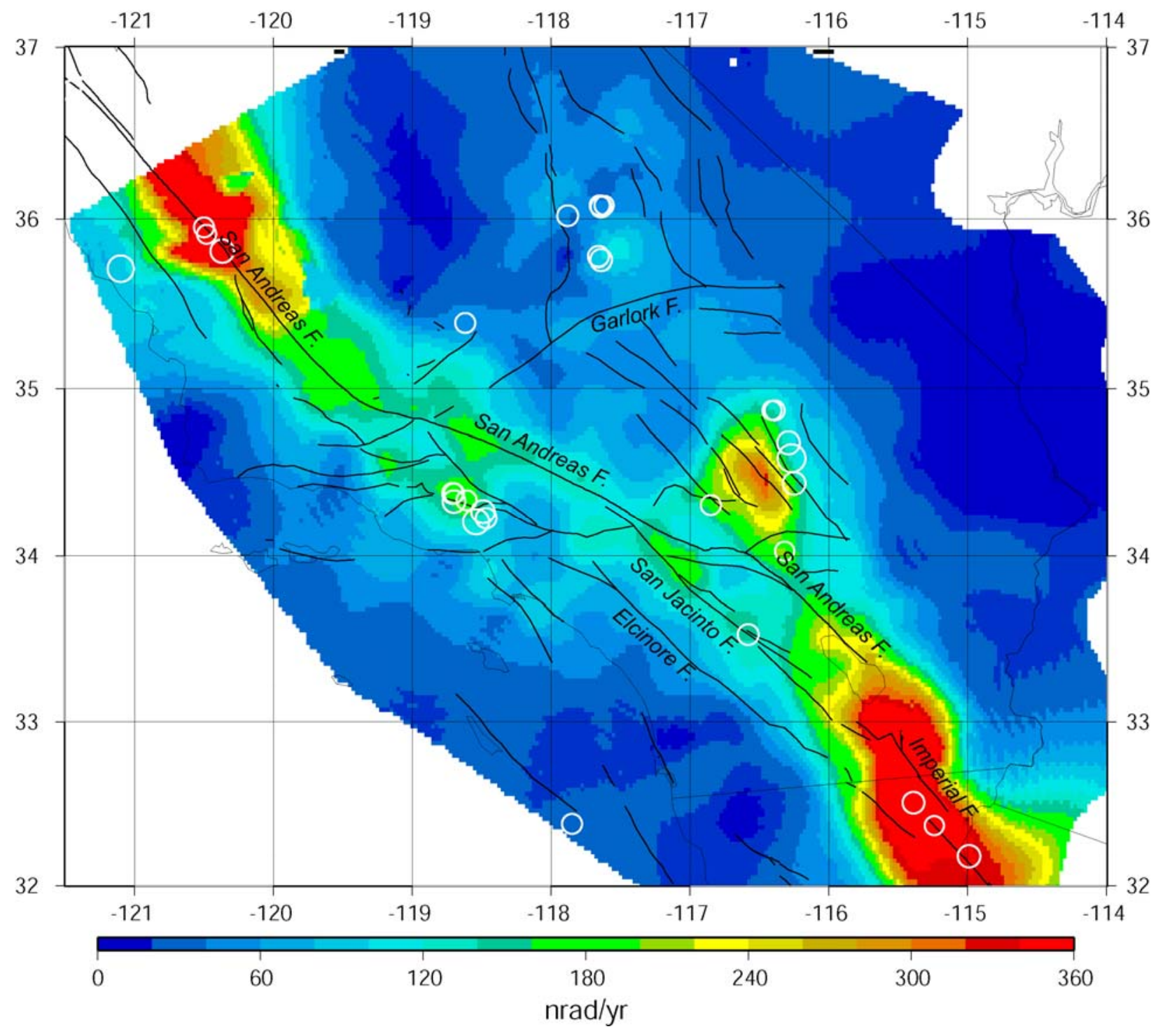


Figure $2 b$

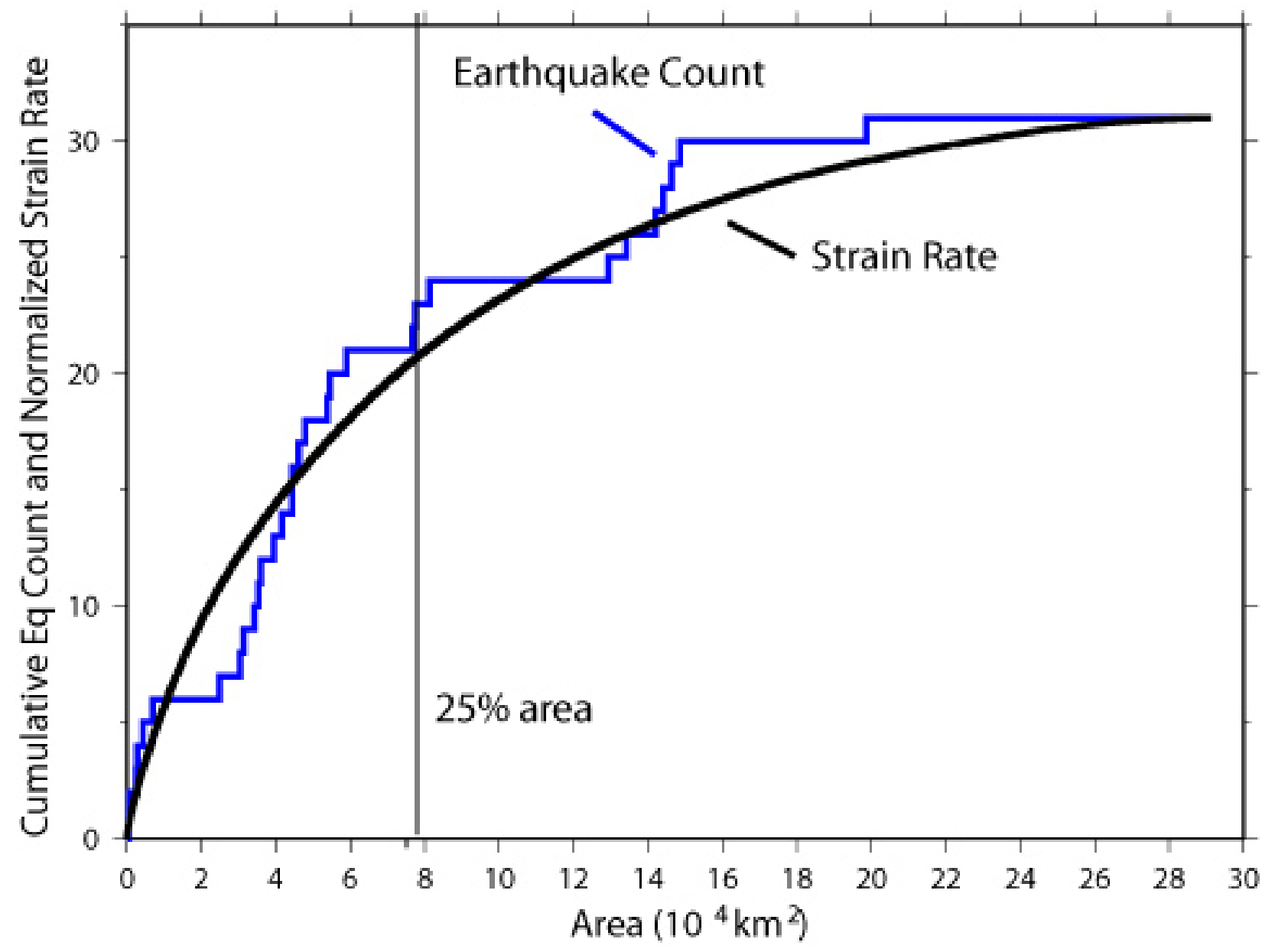

